In which respect this disease resembles poliomyelitis, and incidentally tuberculous meningitis-a disease which often causes a good deal of difficulty in diagnosis.

The protein content of the cerebro-spinal fluid rarely shows any marked increase, whilst the reverse is the case in meningitis, but 0.03 per cent. is sometimes met with. The chlorides are not altered-another point which in a doubtful case may prove of use in making a diagnosis of encephalitis as against meningitis. In the latter the chlorides constantly show a small reduction. Any figure below 0.68 per cent. would suggest meningitis very strongly. So, too, with the glucose content. In encephalitis this is unaltered, or even slightly increased, whilst in cases of meningitis, and more particularly tuberculous meningitis, the quantity is substantially diminished or absent. Changes in the blood are not striking-a small leucocytosis with a total count of 10,000 to 12,000 may be expected.

\section{Differential Diagnosis.}

Encephalitis may be extremely easy or impossible to diagnose. Often it is much easier to recognize from its sequels than during the initial stages. Whilst it is true that in many cases the disease has escaped recognition, it is no doubt also true that in the interest aroused by the advent of a new disorder-at any rate new within our experience-many cases have been labelled encephalitis lethargica which were nothing of the kind. Of acute infections, those most likely to cause trouble in diagnosis are influenza and tuberculous meningitis. The former suggests encephalitis in those cases in which headache, prostration, drowsiness, and fever are the prominent symptoms, whilst the latter may exactly reproduce the clinical picture of encephalitis.

The absence of such signs as retraction of the abdomen, rigidity of the neck, Kernig's sign, and tache cérébrale should help in the diagnosis, though the intrinsic value of each as a diagnostic sign is slight. A careful comparison of the cerebro-spinal fluid in conjunction with the clinical picture should help materially in the recognition of tuberculous meningitis and make the diagnosis certain if other forms of meningeal inflammation are present.

I have already referred to possible difficulties in connexion with chorea, and to errors which occasionally arise in connexion with abdominal pain and rigidity. Vascular lesions may cause great difficulty, and I think that in connexion with these the diagnosis of encephalitis is more likely to be made in error than the converse. The age of the individual is no help in this connexion, as we have seen that encephalitis has no regard to age. Tumour or abscess of the brain sometimes suggests encephalitis, bnt the presence of papilloedema of a marked type would be much in favour of tumour, although we have seen that a low-grade papilloedema sometimes occurs in encephalitis. The absence of any probable cause for abscess should help in its exclusion, but the presence of an ear discharge, present or recent, does not exclude the possibility of encephalitis.

\section{Prognosis.}

Even more difficult than diagnosis is prognosis. Two points have to be considered: will the patient recover, and, if so, will recovery be complete? or will one or other of the sequelae arise? The answer to the first question may remain long in doubt. No definite answer can possibly be given to the second.

Severity of onset does not necessarily imply a fatal issue, nor should too optimistic a view be taken with regard to a case of insidious and apparently mild onset. It would be possible to quote many cases to illustrate the truth of this. Cases with a prolonged febrile period are, I am sure, dangerous. I have referred already to the ominous significance of a slowly rising temperature after a febrile period of two or three weeks. Statistics of the mortality of the disease have varied from 30 to 50 per cent.-figures which are, of course, quite useless in helping to make a prognosis in any individual case.

With regard to sequelae, one can say that of the cases which recover perhaps 50 per cent. will have residual symptoms, from some of which, notably the Parkinson syndrome, they may die. Many of these sequelae no doubt will be slight, and not in themselves disabling. It is inportant to remember that a case which has made an apparently perfect recovery may develop some sequel at any time in the next two years. After that period I should say the risk was negligible, and the majority of patients show evidence of incomplete recovery within twelve months.

\section{TreatMent.}

There is unfortunately no specific treatment that has proved its value. I an convinced of the importance of recognizing the early ambulatory type of the disease and putting the patient to bed. There treatment on general lines must be carried out in so far as diet and regulation of the bowels are concerned. Most of the patients suffer with obstinate constipation.

A gargle of potassium permanganate may well be used by the patient, if he is in a condition to so do-and by those in contact with him.

With regard to any special therapeutic measures, it is safe to say that none has so far offered convincing evidence of success. Hexamine has had an extended trial, by tho mouth, hypodermically, and intravenously, but the results have been disappointing. In some cases serum, either from the patient, or simple horse serum, and in some cases antimeningococcal serum, has been used apparently with advantage. Repeated lumbar puncture has been recommended, although it is difficult to see on what logical grounds; I have, however, seen an improvement in the depth of the lethargy follow a lumbar puncture. Insommia, delirium, or excitement can be dealt with on ordinary lines. The residual symptoms or sequelae are extraordinarily resistant to every therapeutic measure.

\section{THE ANTE-NATAL CLINIC.* BY \\ GUY BELLINGHAM SMITH, M.B., F.R.C.S., SENIOR OBSTETRIC SURGEON, GUY'S HOSPITAL.}

I Have chosen the subject of the ante-natal centre, not because $I$ am the best fitted to deal with it, but as an act of atonement for a certain lack of faith in the first instance. Those of you who, like myself, have worked for rears amongst the very poor know how, with their poverty, goes a certain pride, which resents interference in their affairs. I thought of the difficulties we should have in getting the confidence of these people, and $I$. was unable to anticipate the results that have followed the working out of the scheme. I was reckoning without the enthusiasm and tact that sisters and health visitors have brought to the work, and the enthusiasm again and knowledge that those responsible for the medical side have shown.

I am surprised sometimes at reading the statistical results of fifty years ago, and we occasionally come across to-day despondent remarks as to the progress made in midwifery. These are mainly based on mortality results, and more particularly on those relating to puerperal fever. I have no doubts as to the progress made, having watched the results over a period of forty years. To begin with, figures are not always reliable, and we nust remember further that the range of work dealt with to-day is enormously greater than it was at that time, and that mortality results are more accurately stated. When we remember that our armamentarium consisted of a bottle of ergot, often administered too soon, and a Higginson syringe, which was used indiscriminately for the rectum and the aterus, we may wonder that the results were so good as they were.

But, however we may argue about death rates, who can doubt the enormous improvement that has taken place in the morbidity results? Where are the cases of vesicovaginal fistula that were a commonplace in the operating theatre of a few years past? Where are the sequels of severe injuries and sloughing of the vaginal passage, the atresias, the entire loss of the urethra or of the posterior bladder wall? I never see them to-day.

During the last year or two there has been a complete revolution in the hospital practice of midwifery. For many

* The Purvis Oration, delivered before the West Kent Sedico-Chirurgical Society, December 12th, 1924 . 
years after I started work it was a case of " alarums and excursions," almost invariably at night. Nothing was foreseen. Operations were extemporized in the bedrooms of the poor. I do not know that it was, but it ought to have been, one long nervous strain.

Gradually some improvement came when hospital beds were provided for urgent cases, and we managed to get the more severe cases of pelvic deformity to attend earlier for adequate arrangements to be made. This diminished greatly the number of cases in which the cephalotribe was used. To-day one has peace of mind. Nearly everything is foreseen; very little is left to chance. The extern goes to the patient with a complete history of his case. The cephalotribe and the high forceps will soon, I hope, bo a matter of past history.

I will describe shortly the working of the Salomon Infant Welfare Centre at Guy's Hospital, the only one of which I have any knowledge.

In 1918 the hospital received from the executors of the late Mr. Leopold Salomon $£ 10,000$ to endow an infant welfare centre, and on receipt of this money a committee, composed of representatives of the governors, the hospital staff, and outside persons interested in infant welfare, was appointed, and as a first step took over three houses adjoining the hospital, and by their conversion provided accommodation for ante-natal and post-natal clinics, and for the residence of a sister superintendent, six health visitors, and a domestic staff. The medical staff consists of the two obstetric assistants who are responsible for the antenatal clinics, and two medical officers who are responsible for the post-natal treatment of the mothers and for the welfare of the children up to the age of 5 years.

I like to mention the names of the two men who during these six years have organized the ante-natal work, who have made this department the success that it undoubtedly is, and who will no doubt bring before you one day something more solid than the scraps I bring with me. They are Mr. McNair and Mr. Cook, and to them I owe a great deal that this paper contains. The area of the ante-natal clinic is that of the old maternity district, which extends about a mile round the hospital.

The procedure is shortly as follows. Pregnant women living in the maternity district apply to the almoner of the hospital for a card entitling them to attendance by the hospital during their confinement. Their circumstances having been inquired into, and it being found that they are eligible for treatment, they are referred to the antenatal clinic, where they undergo a thorough examination by the medical officers. Attention here is paid not only to the complications directly due to pregnancy, but also very particularly to such conditions as heart trouble, phthisis, the teeth, and the health generally.

Great stress has been laid upon the teeth, the condition of: these in poor people leading to much anaemia and debility. In consequence a special dental clinic has been instituted under the care of a special dental officer, to whom expectant mothers are sent to have their teeth seen to. We are satisfied that an enormous improvement in health has resulted, the patients becoming better nourished and better able to nurse the children. The mothers attend at regular intervals, according to instructions, up to the time of the confinement, the number of these visits depending naturally upon the presence of some abnormality relating to the pregnant condition or to the general health. In 1923, 2,210 mothers attended during the year, with a total number of visits of 6,773 . It will be seen that this represents a large out-patient department, and that it throws a lot of work on my junior colleagues. Not only are the cases seen here, but they are referred when necessary to the different departments of the hospital for special opinions and advice, some of them being admitted either to the obstetric or to the medical wards.

I referred at the beginning of this paper to difficulties that I anticipated. But I underrated the importance of the hospital connexion. For a long period the district surrounding the hospital has depended on the maternity charity for its confinements, and in this way has grown up a trust in us that was stronger than I realized. It may be some such faith in us that has led to the centre being known in our district as "Salomon's welcome centre."
A most important factor, again, in bringing expectant mothers to us early in their pregnancy is the fact that they have to apply for a card for their confinements. As I have pointed out, after the card is given they are sent to the ante-natal centre, thus enabling us to examine and keep a watch, during their pregnancy, on all cases that we shall subsequently attend at their confinements. The most important details of these examinations, with any possible difficulties likely to be encountered during labour, are entered on a card, which the student or pupil midwife takes with him or her to the case. It is difficult to exaggerate the importance of this procedure.

Another difficulty I expected was due to competition, mainly that occurring as the result of the Midwives Ast. The better class artisan was, I thought, likely to depend on the local midwife. Apart from the long tradition of dependence on the hospital, the poverty of the neighbourhood has helped us, by eliminating largely competition by midwives. Attending the hospital, not only for their confinements but also for all other ailments, the idea of anything like compulsion does not seem to have arisen, and they readily accepted new measures.

Not only has the hospital as a centre helped us in establishing our ante-natal and post-natal clinics, but it affords us another great advantage over clinics not so situated. I refer to the ease with which patients can be sent to the various departments of the hospital for further advice.

As examples of assistance that has been of the greatest use to us I may mention that obtained in the tuberculosis department from Dr. Marshall, and Dr. Hunt's advice with regard to heart cases. I will refer to these again. The facility with which chenical and bacteriological examinations can be made must be mentioned, and also the ease with which any accessory department, such as the dental, can be set up; and last, but not least, the clinic provides invaluable scope for the teaching of students and for research. Whilst the hospital has been of use to the welfare centre, there can be no doubt as to the importance that this centre has been to the obstetric staff of the hospital. The two have helped one another, and through this close association great improvement in the treatment of women during pregnancy and labour has resulted.

Serious operations during the course of and often late in labour, that at one time occurred with comparative frequency, and had to be dealt with as matters of urgency, are now anticipated, and those that are likely to be attended with difficulty are admitted to hospital before labour begins. The importance of this in avoiding any clelay in operative procedures is obvious, and not only is unnecessary maternal suffering avoided, but a reduction both of the death rate, and more particularly of ensuing morbidity of mother and child, results. Dr. Eason, the superintendent of Guy's Hospital, in a paper describing the institution and working of the Salomon centre, from which I have most unblushingly stolen what I wanted, concludes with the following paragraph:

" The experience which the hospital authorities have derived from the establishment of the infant welfare centre makes it quite clear that infant welfare centres should, if possible, be closely associated with, or actually established in, hospitals, and that infant welfare centres should in all cases, if possible, include ante-natal clinics, so that the mother and the child can be kept under continuous observation by the same set of medical officers."

In addition to being seen at the infant welfare centre the mothers are visited at their homes by the health visitors, who give them advice as to domestic hygiene and the feeding of the infant. To show the importance of their assistance to the mothers $I$ will only mention the fact that owing to their encouragement 86 per cent. of the women feed their babies at the breast.

It is too early yet to give anything like a statistical history of results, but as seven out of every ten people seen at the centre have been seen before, and as notes relating to them are kept, we should in time have invaluable material.

There are one or two subjects I. should like to refer to shortly, and the first of these is heart disease complicating pregnancy. This has interested me considerably during the last two or three years. As showing the importance of the subject, approximately 200 cases have been seen since the opening of the centre. Only a small proportion of these 
are serious enough to admit to hospital, the majority going through pregnancy and labour without serious symptoms or risk. It will be interesting to see as time goes on what happens in subsequent labours.

There are a few cases, however, in which the heart is so seriously affected that one anticipates a fatal result, either during labour or shortly afterwards. There is no doubt at all as to the serious effect on the heart of prolonged efforts at expulsion. I used to be asked to see these cases with a view to induction of labour. But $I$ rarely agreed to this. If anything, induction is a more serious trial than labour at term, and I hold the view that those patients who would survive induction would pass equally well through labour at full time, and that those who appeared likely to die in the course of labour were equally likely to die as the result of induction.

I think it was with Dr. French that I first discussed the question of Caesarean section in these cases, over a case of mitral stenosis and auricular fibrillation. From her condition it seemed abundantly clear to both of us that the patient was not likely to survive a labour lasting some hours. I do not think Dr. French imagined that she would stand a Caesarean section, but in any case he thought it would cause less distress. I was more hopeful. I had had some experience in abdominal sections of cases with very bad hearts, and I knew that, provided they were not asphyxiated, they took anaesthetics and stood the operation better than might be anticipated. I found it to be the same in this case. She gave no particular anxiety either during the operation or subsequently, and although when she left the hospital her heart was still very irregular, she was able to walk about without marked dyspnoea. Since then I have performed Caesarean section on a few more cases after consultation with Dr. Hunt.

One case that suffered from paroxysmal tachycardia had severe mitral and aortic disease. Beyond a little bronchitis she did well after the Caesarean section, and suckled the baby. A month later she had an attack of confusional insanity, and ten months later was again admitted to hospital, when she died.

It appeared to me that the very bad cases suffered mainly from mitral stenosis and auricular fibrillation, and, having a talk recently with Dr. Hunt, I asked him what conditions he considered would cause the gravest anxiety as regards labour. $\mathrm{He}$ thought that cases with auricular fibrillation, very large heart, and those having very bad exercise tolerance would give the worst prognosis. As regards anaesthetic, C.E. was given with oxygen. The most important thing is to avoid asphyxiation and anything like overstimulation of the heart. I think Dr. Hunt has been as much struck with the results as I have been. The relief to the mothers has been much greater than either of us could have anticipated, and recovery has been speedy. Instead of twenty-four hours, perhaps, of the greatest distress during labour, with death hanging over their heads-and I have little doubt that few of the cases I have operated upon would have survived this ordeal-there has been comparatively little distress for the patients and very little anxiety for those who had to look after them.

Only recently $I$ had a patient sent to me with a history that she had nearly died during her last confinement, that she had adopted precautions against further pregnancy, which, as generally happens in such cases, had failed. She was four to five months pregnant. Dr. Hunt considered that pregnancy should be terminated in the interests of the patient, who was rapidly becoming worse. Induction at this stage of pregnancy is often a tedious business, requiring more than one anaesthetic, and as opening the abdomen allowed me to sterilize her, I did a subtotal hysterectomv. She gave us not a moment's anxiety, which we can usefully compare with her narrow escape at the previous confinement. The results have been such that $I$ shall not hesitate in any future case in which the risks of labour appear to be such that death is likely to result to perform as an alternative Caesarean section.

Albuminuria.-Finding albumin in the urine at an early , tage in pregnancy, we ought theoretically to avoid the risk of eclampsia. We have not done so. Out of 4,000 cases in the last two years we have had one case. This was a patient who, suffering from chronic nephritis and a high blood pressure, had in the course of her first pregnancy a stillbirth at seven months.

She was admitted during her second pregnancy at about the twenty-eighth week for albuminuria. This improved so much that she was allowed to go home. She did not carry out the instructions given her, and she came back in a week with eclampsia. The Dublin treatment was employed, but failed to check the fits. Caesarean section was then performed, and the patient recovered. The failure in this case was clearly due to the patient, who, probably tired of the rigid treatment as regards diet endured for some time, allowed herself a little more licence than was good for her. This will probably always prevent us getting those theoretically perfect results I have mentioned above.

The only criticism I would make on the treatment above is this: that $I$ dislike Caesarean section as a late measure when other methods have failed. It has brought it into discredit in the past, and although it succeeded here it generally fails. The rexed question of Caesarean section versus the Dublin treatment we still have to leave to the future to decide.

Phthisis as a complication of pregnancy presents considerable difficulties. Roughly one can say that whilst some cases became rapidly worse, in others no alteration was perceptible, whilst in a few there seemed to be some improvement even in the general condition.

This vague position has been cleared up for me by Dr. Marshall, and from some notes on the subject he has kindly made for me I select the following. It is in early cases of phthisis that pregnancy produces an exacerbation of the disease. In these early cases the general condition of the patient remains good during the first few months of the pregnancy, and symptoms may even be ameliorated. Deterioration, with pyrexia, wasting, and increased cough, often commences about the seventh month of pregnancy, and there is rapid extension of the disease after childbirth.

A number of cases have been seen during pregnancy who had had pleural effusion or open pulmonary tuberculosis at an earlier date, but in whom the disease had been arrested. With careful supervision most of these patients have gone through their pregnancy and puerperium without any ill effects on the lungs. In a few there has been a lighting up of the old disease, though in most of these cases it has become inactive again under treatment. When the phthisis is of some years' standing and the patient does not show severe toxaemia pregnancy does little harm. There may be a slight increase in pyrexia and sputum at the latter end of pregnancy, but as a rule there is no gross deterioration in the general condition.

It is clear from these remarks of Dr. Marshall's that the cases that call for interference are the early ones, the arrested and chronic cases being allowed to go to full term, rest and extra nourishment being the most important details of treatment. The mother is allowed to nurse her baby if there is no toxaemia. Dr. Marshall holds that the baby is more robust and better able to withstand infection if it is allowed to take its natural food.

For some years past cases have been sent to me from time to time in the early stages of pregnancy, to have this terminated by the induction of miscarriage. It was not long before I discovered that this was a useless procedure. The patients of the hospital class invariably returned, in a few months' time, again pregnant; and even in people of a better class who had been warned of the risks involved, and who had taken measures of precaution against conception, it was by no means uncommon to find that pregnancy had recurred. As a result I refused for a time to entertain any idea of induction.

Another thing I found, which I am glad to see Dr. Marshall agrees with, is that induction either of miscarriage or labour seemed to do as much harm to the pulmonary condition as allowing the pregnancy to go to full term. I an not at all clear as to the reason of this.

As a result of all this $I$ adopted another line of treatment. When it was made clear to me that not only should the existing pregnancy be terminated but that it would be unsafe for the patient to have any children at a future date, I opened the abdomen and emptied the uterus through the abdominal route, sterilizing the patient by removal of the Fallopian tubes. In early cases, in place of this, removal of the uterus by supravaginal hysterectomy can be 
considered. Here again, as in heart discase, I was pleased to find that the results were better than anticipated, and attended with less ill effects than appeared to attend induction of miscarriage and labour. Dr. Marshall, who sees these cases over a long period of time, is so satisfied with the results that he states definitely "that the best results are obtained by Caesarean section" (meaning by this the emptying of the uterus by the abdominal route at any time of pregnancy).

I think the number of cases in which an operation is likely to be called for is small, and I still feel that interference in any case should be most carefully considered before it is undertaken.

I have already mentioned that cephalotripsy is becoming an operation of the past. Curiously enough, we have found that when obstructed labour occurs calling for destruction of the infant it is more likely to be found in a multipara than in a primipara. Every now and again a woman of about 40 who has had a series of children born without mishap gets obstruction. This has been explained by saying that the children get progressively larger. I know no very satisfactory evidence of this. It may be due to falling forwards of the sacral promontory owing to increasing weight of body, and possibly to working hard shortly after confinements, when the pelvic joints are still lax. My colleague Mr. McNair suggests that it is due to lack of play in the pelvic joints during labour, the joints becoming ankylosed. We have had one such case recently, in which we performed Caesarean section.

Time does not allow me to go into the question of contracted pelvis. I might, however, conclude with a few remarks on the subject of induction of labour. If one were to classify obstetricians it would be into those who like incluction (or say they do) and those who frankly do not. I put myself in the second class.

In theory nothing is simpler. For some reason or another it is decided to induce labour at the thirty-sixth week. When this time arrives a few bougies are introduced into the uterus, and in twenty-four hours labour starts and the child is born without further anxiety. This assumes two things-that we know exactly when the thirty-sixth week has arrived, and that the infant is going to remain the same in size as on the preceding occasion. These assumptions being fallacious, we have to fall back in addition on the rather difficult procedure of estimating the relative sizes of the child's head and the pelvis.

In some cases we find ourselves inducing earlier than $\pi \theta$ intended, and possibly lose the child from prematurity; in others we have a shrewd suspicion that induction has coincided with labour at term. In one case labour comes off with such ridiculous ease that we wish we had waited for labour at full term; in another we meet with more difficulty than we anticipated, and perhaps lose the child.

Further, instead of labour coming on shortly after the introduction of bougies, it often hangs on for a week or more, to the anxiety of the doctor, the discomfort of the patient, and the mental distress of the relatives, who will not be convinced that everything is right. This operation is, as far as I can gather, an essentially English one, and perhaps the most uncertain and uncomfortable one we possess.

The margin of cases suitable for induction is very small and none too easy to detect. Perhaps its most important indication is in post-maturity. I have always preferred, where possible, to go to full term. It is curious in what a number of cases in which one was tempted to induce labour, the pregnancy is terminated without undue diffculty. In the few remaining one can wait till labour starts, if necessary, and, satisfactory progress not being made, perform Caesarean section; and I do not believe that many of these cases would have proved satisfactory if induction had been performed earlier as an alternative

As I remarked, I am in a position at the present time to make a few scattered remarks only, and these mainly in reference to recent experience in heart and lung cases. I feel certain, however, that the establishment of these centres has already proved of very great value to us, and that they will enable us in the future to work out the many difficult problems that still confront us in obstetric practice.

\section{THE THYROID AND MANGANESE TREATMIENT IN VARIOUS DISEASES. (First Report.)}

BX

\author{
HERBERT W. NOTT, M.R.C.S., L.R.C.P.,
} LITTLE SUTTON, BIRKENHEAD.

Is the course of work as a general practitioner cases are constantly encountered, due to some toxaemia or chronic infection which one tries in vain to remove. I noticed that many of these chronic cases presented the signs of thyroid inefficiency, and was thus led to study Colonel McCarrison's well known books, The Thyroid Gland in Health and Disease and Studies in Deficiency Diseases. As the idea of this treatment was evolved entirely from this study, whatever merit may in the future be found to pertain to it will be due to the vast amount of work he has done.

The treatment consists in the administration of rectal injections of a solution of pure potassium permanganate, of various strengths, given at various intervals, according to the severity of the infection or the length of time it has existed. The standard solution used is 1 grain of permanganate to $1 \frac{1}{2}$ pints of sterile warm water, and, as evidence is accumulating that the tablets or soloids of the manufacturing chemists are more reliable than are the ordinary commercial crystals of the drug, it should be made from these. As the oxidizing power of potassium permanganate is somewhat reduced by boiling water, a pellet of the drug is crushed to powder in an earthenware basin with a teaspoon or some metal crusher; cold water is poured on to it, and hot water is added to the required volume and temperature. There should be no undue delay in using the preparation, as it is believed reduction in its power takes place fairly quickly on exposure. A metal or painted basin should not be used.

An injection is given with the patient lying on his left side, and it must be administered slowly by means of a funnel and tube or Higginson's syringe. It should be given after the bowels have acted; it should not be used to effect the purpose of an ordinary enema, as it is given with the intention of its being retained.

Patients sometimes have to be encouraged during the first few days, but as they soon feel better they are willing to continue. When subjects of stasis are beginning the treatment, a larger volume of the solution is needed during the first few dass until the bowels are cleared. Doses of liquid extract of cascara are given two or three times a day to cultivate an action of the bowels every twelve hours. As the neuro-muscular functioning of the bowel improves, it is more readily stimulated, so that smaller doses of the liquid extract are soon found to be sufficient, until, by degrees, none is required. Those who have difficulty at first in retaining even a small quantity of the solution should remain lying down for twenty minutes or half an hour, when the desire to return it will have passed off. Or, by assuming the knee-elbow position, the fluid runs higher up the bowel, and the desire to void it passes away completely in most cases.

The quantity and the strength of the solution to be used in any given case can only be estimated by experience and by studying the individual. For instance, two doses of a quarter of a pint in the day may be found more effective than one close of half a pint. In every case it is necessary to warn the patients that they may feel some pain in the epigastrium within a few minutes after the injection is given. It does not last long as a rule, and it is not often complained of after the first few days. They should also be warned that, from three days to two weeks after treatment has commenced, they mav pass long white skins or strings of mucus in the stools. Unless this warning is given some may be frightened, thinking they are suffering from tapeworms. Complete mucous casts of the bowel from three to fifteen inches in length are sometimes passed. Enmeshed in these casts enteroliths, and perhaps grape skins, etc., may be seen occasionally.

Thyroid extracts in compressed form, as supplied by most manufacturers, are now very reliable, and these are 